Guillermo Alberto Pérez Fernández*

Departamento de Cardiología, Servicio de Cardiología Clínica, Hospital Celestino Hernández Robau, Santa Clara, Villa Clara, Cuba

* Autor para correspondencia. Maceo N. ${ }^{\circ} .263$ e/San Miguel y Candelaria. Santa Clara. Villa Clara. Cuba.

Teléfono: +53(42)204294.

Correo electrónico: gpfholy@gmail.com https://doi.org/10.1016/j.acmx.2017.08.005

1405-9940/

(c) 2017 Instituto Nacional de Cardiología Ignacio Chávez. Publicado por Masson Doyma México S.A. Este es un artículo Open Access bajo la licencia CC BY-NC-ND

(http: //creativecommons.org/licenses/by-nc-nd/4.0/).

\section{Síndrome de Kounis}

\section{Kounis syndrome}

\section{Introducción}

El síndrome de Kounis (SK), descrito por Kounis y Zavras en 1991, es una urgencia médica probablemente infradiagnosticada, en la que concurren un síndrome coronario agudo en forma de angina inestable, vasoespástica o no, e incluso un infarto agudo de miocardio, a causa de una reacción alérgica o de hipersensibilidad, habiéndose descrito casos incluso en anafilaxia $^{1-3}$. Es importante reseñar que las reacciones alérgicas graves pueden ser causa de síndrome coronario agudo en pacientes con coronarias sanas y sin factores de riesgo cardiovascular.

\section{Caso clínico}

Presentamos el caso de un varón de 56 años, con antecedentes de obesidad y dislipidemia, que acudió a Urgencias de atención primaria por prurito generalizado y lesiones cutáneas evanescentes de 5-6 h de evolución. Estando en la sala de espera del mismo, el paciente presentó cuadro de mareo, sudoración y dolor epigástrico irradiado a espalda, presentando posteriormente un cuadro sincopal con relajación de esfínteres e hipotensión arterial asociada. No presentó convulsiones, mordedura de lengua, dolor torácico ni palpitaciones. Se realizó un electrocardiograma (EKG) que mostró un ritmo sinusal a $55 \mathrm{lpm}$ con elevación del segmento ST de V2-V6 de hasta $2 \mathrm{~mm}$, motivo por el que se activó al servicio de Emergencias (112) y se remitió al paciente al servicio de Urgencias de nuestro hospital.

A su llegada el paciente refirió disminución del prurito con la medicación administrada (Polaramine $5 \mathrm{mg}$ y metilprednisolona $80 \mathrm{mg}$ ). Se encontró asintomático desde el punto de vista cardiovascular, normotenso, sin dolor torácico, palpitaciones ni disnea. Además, el EKG reflejó una normalización de las alteraciones anteriormente descritas. El paciente no refirió ingesta previa de ningún fármaco ni uso de ropa nueva y/o geles/cremas. Refirió haber salido a correr por la misma ruta que realizaba habitualmente, no recordando picadura de insecto ni contacto con ningún material atípico. El paciente no presentaba ningún antecedente de interés ni se encontraba bajo ningún tratamiento farmacológico.
En la exploración se observaron lesiones habonosas de aspecto urticarial en hombros, flanco izquierdo y cuello; lesiones que desaparecieron al cabo de $2 \mathrm{~h}$, con aparición de lesiones similares en mesogastrio y dorso de ambos pies, siendo el resto de la exploración física, incluyendo la presión arterial, normal.

En los análisis de laboratorio, incluyendo la seriación de enzimas cardiacas, no se encontraron alteraciones significativas. Se realizó una radiografía de tórax en la que no se objetivaron hallazgos de interés. En los diferentes EKG realizados se observó (a) EKG realizado en Urgencias del centro de salud: ritmo sinusal a $55 \mathrm{lpm}$, elevación de punto J V2-V6 hasta $2 \mathrm{~mm}$; (b) EKG realizado a su llegada al servicio de Urgencias hospitalarias: ritmo sinusal a $54 \mathrm{lpm}$, corrección de la elevación de punto J; (c) EKG realizado posteriormente: ritmo sinusal a $57 \mathrm{lpm}$, T aplanada en $\mathrm{V} 1$, $\mathrm{T}$ negativa en $\mathrm{V} 2$, resto similar al previo. Se realizó además un ecocardiograma v-SCAN en el que la única alteración destacable fue una insuficiencia mitral ligera. Durante su hospitalización en Cardiología, se realizó un ecocardiograma transtorácico, que no evidenció cardiopatía estructural, así como un angioTAC coronario, que mostró una placa calcificada periférica en segmento proximal de DA y una placa fibrolipídica en tercio medio de DA, sin condicionar ninguna de ellas estenosis significativa (24 y $25 \%$, respectivamente).

Con todo ello, nuestro paciente fue diagnosticado de episodio de anafilaxia, sin claro desencadenante y episodio de síncope y de ascenso transitorio de ST en cara anterior (SK tipo I) relacionado con previo. Al alta, el paciente recibió una cita en Alergología para realizar un estudio alergológico completo.

\section{Discusión}

La historia clínica es fundamental para llegar al diagnóstico de SK, ya que no existen protocolos clínicos establecidos para confirmar o excluir su diagnóstico ${ }^{4}$. Debemos objetivar una clínica simultánea tanto de reacción alérgica aguda (rash, urticaria, angioedema, disnea, hipotensión, náuseas) como de un evento coronario agudo (malestar general, dolor torácico de características anginosas, cortejo vegetativo $)^{5}$.

Se trata de una entidad causada por el efecto de mediadores proinflamatorios liberados masivamente por los mastocitos en el tejido del corazón, arterias y placas coronarias. Debido a que una serie de estímulos pueden desencadenar la desgranulación de los mastocitos, 
1. Monitorización del paciente

2. Evaluación cardiológica y tratamiento del síndrome coronario agudo

- Nitroglicerina

- Evitar B-bloqueantes

- Evaluar bloqueadores de canales de calcio (verapamilo,diltiazem)

- Double antiagregación (tipo II y III)

- Intervención coronaria percutánea-ICP-(tipo II y III)

3. Tratamiento de emergencia de una reacción alérgica

- Eliminar el desencadenante alérgico

- Adrenalina im

- Oxígeno

- Resucitación con fluidos intravenosos

- Glucocorticoides

- Anti-H1

Figura 1 Tratamiento del síndrome de Kounis.

Fuente: modificado de Fassio et al. ${ }^{6}$.

principalmente reacciones alérgicas mediadas por IgE, es concebible que cualquier reacción alérgica podría facilitar el espasmo coronario o la disrupción de la placa. Los mediadores inflamatorios, incluyendo histamina, proteasas neutras, productos de ácido araquidónico, factor de activación de plaquetas y una variedad de citoquinas y quimiocinas, aumentan en sangre $u$ orina tanto en episodios alérgicos como en síndromes coronarios agudos. Parece existir una vía común entre síndromes coronarios alérgicos y no alérgicos. Hoy en día hay evidencia de que los mastocitos no solo acceden a la región culpable antes de la erosión o rotura de la placa, sino que liberan su contenido antes de un episodio coronario $\mathrm{real}^{3}$.

No está establecido qué determina que se desarrolle un evento coronario, ya que no todos los pacientes que presentan una reacción alérgica lo desarrollan. Se ha propuesto que existe un nivel límite de la activación de mastocitos y liberación de mediadores por encima del cual se desarrolla el espasmo coronario o la rotura de la placa de ateroma ${ }^{4}$. Dicho límite estaría íntimamente asociado al lugar donde ocurre la reacción antígeno-anticuerpo, el área de exposición, la liberación de mediadores $y$, por supuesto, la gravedad de la reacción alérgica.

Numerosos agentes se han implicado como desencadenantes de este síndrome: fármacos (betalactámicos y antiinflamatorios no esteroideos principalmente), alimentos, picaduras de insectos, contrastes o exposiciones ambientales $^{2}$. Actualmente se conocen tres tipos de SK: tipo I (pacientes con coronarias normales, donde las reacciones de hipersensibilidad producen un espasmo coronario), tipo ॥ (pacientes con enfermedad aterosclerótica, donde el espasmo produce rotura de la placa y trombosis) y tipo III (en relación con trombosis en pacientes con stents farmacoactivos $)^{2}$.

El SK es una manifestación coronaria aguda compleja que requiere decisiones de tratamiento rápidas, dirigidas no solo a la vascularización del miocardio, sino también al tratamiento de la reacción alérgica concomitante. A día de hoy, no existen guías para el tratamiento de este síndrome, pero, en general, estos pacientes son subsidiarios de tratamiento con antihistamínicos, corticoides y antitrombóticos ${ }^{4}$. En una revisión publicada en 2016 se ha propuesto un esquema terapéutico para el SK (fig. 1) ${ }^{6}$. En pacientes jóvenes y sanos, el tratamiento de primera línea debería incluir agentes vasodilatadores (nitratos y antagonistas del calcio). En pacientes con SK tipo ॥ debería seguirse el protocolo del síndrome coronario agudo $^{7-9}$.

El SK se describe cada vez con más frecuencia en la práctica clínica, por lo que es importante ser conscientes de su existencia. Nos encontramos ante una enfermedad única, en la que debemos pensar cuando el dolor torácico agudo se acompaña de síntomas alérgicos y cambios electrocardiográficos.

\section{Bibliografía}

1. Molina Anguita M, García Rodríguez C, Méndez Díaz Y, et al. Síndrome de Kounis, urgencia diagnóstica. Semergen. 2016;42:e133-5.

2. Garcipérez Vargas FJ, Mendoza J, Sánchez-Calderón P, et al. Shock cardiogénico secundario a síndrome de Kounis tipo II inducido por metamizol. Rev Esp Cardiol. 2012;65:1134-42.

3. Kounis NG. Kounis syndrome (allergic angina and allergic myocardial infarction): A natural paradigm? Int J Cardiol. 2006;110:7-14.

4. García Núñez I, Algaba Mármol MA, Barasona Villarejo MJ, et al. Kounis syndrome after levofloxacin intake: A clinical report and cross-reactivity study. J Investig Allergol Clin Immunol. 2016;26:335-6.

5. Rico Cepeda P, Palencia Herrejón E, Rodríguez Aguirregabiria MM. Síndrome de Kounis. Med Intensiva. 2012;36:358-64.

6. Fassio F, Losappio L, Antolin-Amerigo D, et al. Kounis syndrome: A concise review with focus on management. Eur J Intern Med. 2016;30:7-10.

7. Rodríguez Justo S, González Babarro E, García Villar E, et al. Síndrome de Kounis: síndrome coronario agudo inducido por una reacción alérgica. Emergencias. 2011;23:492-500.

8. Almpanis G, Siahos S, Karogiannis NC, et al. Kounis síndrome: Two extraordinary cases. Int J Cardiol. 2011;147:e35-8.

9. Lorca R, Velasco E, Madre J, et al. Kounis syndrome: Identifying the trigger. Int J Cardiol. 2016;209:179-80. 
Eva María García-Atienza ${ }^{a, *}$, José Luis Agudo-Mena ${ }^{\mathrm{b}}$, Yolanda Prieto-Corredor ${ }^{\mathrm{c}}$ y Vicente Ferrer Bleda ${ }^{\mathrm{d}}$

${ }^{a}$ Gerencia de Atención Integrada, Centro de Salud Zona 8, Albacete, España

b Servicio de Dermatología, Complejo Hospitalario Universitario de Albacete, Albacete, España

c Servicio de Urgencias, Complejo Hospitalario Universitario de Albacete, Albacete, España

d Servicio de Cardiología, Complejo Hospitalario

Universitario de Albacete, Albacete, España
*Autor para correspondencia. Teléfono: +618253896; Centro de Salud Zona 8 C/ San Juan n. 3702006 Albacete, España

Correo electrónico: evagatienza@gmail.com (E.M. García-Atienza).

https://doi.org/10.1016/j.acmx.2017.10.005

1405-9940/

(c) 2017 Instituto Nacional de Cardiología Ignacio Chávez. Publicado por Masson Doyma México S.A. Este es un artículo Open Access bajo la licencia CC BY-NC-ND (http: / / creativecommons.org/ licenses/by-nc-nd/4.0/). 